Jinks, J. L. (1959). J. gen. Microbiol. 20, 223-236

\title{
Selection for Adaptability to New Environments in Aspergillus glaucus
}

\author{
By J. L. JINKS \\ A.R.C. Unit of Biometrical Genetics, Department of Genetics, \\ University of Birmingham
}

SUMMARY: Colonies of Aspergillus glaucus arising from single asexual spores or hyphal tips of the same or different homokaryon clones often vary in their adaptability to new environments. This shows itself as differences in the percentage survival and mean lag period of inocula taken from colonies growing on a normal medium when transferred to one containing a poison such as mercuric chloride, or unfamiliar sugar sources, e.g. arabinose, galactose, lactose and xylose.

The differences in adaptability to these new media between homokaryon clones are characteristically nuclear in origin, while those between colonies of the same clone are characteristically cytoplasmic. The nuclear system might be formally regarded as epistatic to the cytoplasmic system involved in these adaptive changes.

Selection for the cytoplasmic differences can produce marked changes in the adaptability of the asexual spores of single colonies, even though the selection technique is such that colonies in the direct line of selected descent are at no time exposed to the new media.

The changes in adaptability to mercuric chloride are independent of those for adaptability to the new sugars and only the latter shows any correlation with changes in rate of growth. There is therefore evidence of two or possibly three cytoplasmic systems with much the same properties of variation but independent in action and transmission.

Adaptations to new environments can involve both nuclear and cytoplasmic changes. In Aspergillus glaucus the adaptive changes directly responsible for growth in the presence of poisons and unfamiliar sugar sources are confined to the cytoplasm (Briault, 1956). On the other hand, genotypes differ in their ability to adapt to these new media. In the present investigations, therefore, we shall examine the interrelations of nucleus and cytoplasm in determining the ability of an inoculum to adapt to a new environment. At the same time comparisons will be made between the properties of the cytoplasmic system involved in the adaptive changes and of that responsible for the naturally-occurring variation in the morphological characters, rate of growth, perithecial density, pigmentation, etc. With these two aims in view we shall extend the approach developed by Jinks (1954, 1956-58) to investigate the latter system to see how far the explanations and methods of investigation are applicable to the adaptive changes and to establish how far the cytoplasmic systems involved are common to both.

\section{METHOD}

Material. The material consists of the wild isolates of Aspergillus glaucus described in earlier papers and various derivatives obtained by introducing major gene markers. The latter, which primarily affect spore colour or colonial 
morphology, have no other significance than that they facilitate the genetic analyses which are carried out from time to time on these isolates to determine the nuclear or cytoplasmic origin of any change in phenotype.

Medium. The basal medium employed throughout was solid Czapek $+5 \%$ sodium chloride. The mercuric chloride was added to this in dosages between $\frac{1}{2}$ and 20 p.p.m. ( $\mu$ g. mg. $/ \mathrm{ml}$.), while the alternative sugar sources galactose, xylose, arabinose and lactose were each substituted for sucrose at the rate of $2 \%$ by $(w / v)$. The mercuric chloride solution, which was freshly made on each occasion it was used, was sterilized independently of the basal medium and only added to the latter just before pouring into the Petri dishes.

Techniques. The majority of propagations and tests were made on samples of single asexual spores which were prepared, counted and plated as described by Jinks (1952) and Pontecorvo (1953).

The percentage survivals of random samples of asexual spores on the new media are given relative to those of a duplicate sample on the basal medium. Similarly, the mean lag periods, which were obtained by averaging the number of days required for the individual asexual spores to produce a microscopically recognizable colony on the new media, are corrected for the time taken for spores of a duplicate sample to produce similar colonies on the basal medium.

Occasionally hyphal propagations were used and unless otherwise stated the inocula contained approximately fifty hyphal growing-tips taken from the periphery of a colony. In one experiment, the results of which are summarized in Table 4, inocula containing different numbers of hyphal growingtips were used. The latter were counted under the microscope for those inocula which contained 4, 16 and 32 intact growing-tips, including in the counts only those tips which had not lost their contents as a result of cutting. The larger inocula, however, were estimated as that sized arc of the periphery of a colony which on the average was found to contain 50, 100, 200, etc., intact growing-tips on counting a duplicate sample.

Phenotypic differences of nuclear and cytoplasmic origin were separated by the heterokaryon test, the various aspects of which have been described in a number of papers (Jinks, 1954, 1956, 1958: Arlett, 1957; Gibson \& Griffin 1958).

\section{RESULTS}

\section{Differences between homokaryon clones}

Homokaryon clones differ in their adaptability to the new environments on both criteria used, viz. the percentage survival of transfers to the new media and the duration of the initial lag period. Examples, using random samples of asexual spores, produced on the normal medium as inocula, are given in Tables 1 and 2. In these tables the percentage survival at various concentrations of mercuric chloride and the mean lag of the survivors are given for three homokaryon clones.

Similar, but on the whole less marked, differences between homokaryon clones were obtained on the new sugars where the survival rates were high and the lag period of short duration. 
These differences in adaptability between homokaryon clones, which of course are found with hyphal as well as spore inocula, can be analysed in such a way as to determine their nuclear or cytoplasmic origin (Jinks, 1954, $1956,1957,1958)$.

Table 1. The percentage of asexual spores in random samples from three homokaryon clones which successfully adapted, i.e. formed colonies on 2, 3.5 and 5 p.p.m. of mercuric chloride

\begin{tabular}{|c|c|c|c|}
\hline Homokaryon: & 2.1 & 2.w. & 1.8 \\
\hline Conc. of mercuric & \multicolumn{3}{|c|}{ Percentage of random sample } \\
\hline $\mathbf{2}$ & $67 \cdot 2$ & $69 \cdot 6$ & $36 \cdot 5$ \\
\hline 3.5 & $51 \cdot 4$ & $62 \cdot 5$ & $19 \cdot 5$ \\
\hline $\mathbf{5}$ & $6 \cdot 8$ & 44.7 7 & $10 \cdot 0$ \\
\hline
\end{tabular}

Table 2. Mean lag period in days of those asexual spores from three homokaryon clones which were capable of growth on $2,3.5$ and 5 p.p.m. of mercuric chloride

\begin{tabular}{|c|c|c|c|}
\hline Homokaryon: & 2.1 & $2 . w$ & 1.8 \\
\hline Conc. of mercuric & \multicolumn{3}{|c|}{ Mean lag in days } \\
\hline 2 & $3 \cdot 32$ & 1.45 & $2 \cdot 87$ \\
\hline $3 \cdot 5$ & $\mathbf{3 \cdot 9 7}$ & $2 \cdot 71$ & $4 \cdot 73$ \\
\hline 5 & $7 \cdot 30$ & 5.91 & $7 \cdot 32$ \\
\hline
\end{tabular}

The technique may be illustrated by reference to clones $2 . w$ and 1.5 (Tables 1 and 2). These two homokaryons were readily distinguishable morphologically both from one another and from the heterokaryon which was easily formed between them. Since the heterokaryon produced a high proportion of homokaryotic spores the two homokaryons could be re-isolated from their heterokaryotic association following a period of co-existence of the two kinds of nuclei in the same cytoplasm. The adaptability of the homokaryons can, therefore, be compared before and after the heterokaryotic association of their nuclei. Table 3 shows the results of one such comparison of the adaptability of homokaryon 1.s before and after heterokaryotic association with homokaryon 2.w. The character is the percentage of asexual spores in random samples which successfully adapt to three different levels of mercuric chloride.

Table 3. The percentage of asexual spores in random samples from homokaryon $1 . s$ which successfully adapted to $2,3.5$ and 5 p.p.m. of mercuric chloride before and after heterokaryotic association with homokaryon 2.w.

\begin{tabular}{|c|c|c|}
\hline \multirow[b]{2}{*}{$\begin{array}{l}\text { Conc. of mercuric } \\
\text { chloride (p.p.m.) }\end{array}$} & \multicolumn{2}{|c|}{ Percentage of random sample } \\
\hline & $\begin{array}{c}\text { Before } \\
\text { heterokaryon }\end{array}$ & $\begin{array}{c}\text { After } \\
\text { heterokaryon }\end{array}$ \\
\hline 2 & $35 \cdot 8$ & $37 \cdot 2$ \\
\hline $3 \cdot 5$ & $19 \cdot 0$ & $20 \cdot 0$ \\
\hline 5 & $8 \cdot 3$ & $11 \cdot 6$ \\
\hline
\end{tabular}


The results summarized in Table 3 were analysed by a contingency $\chi^{2}$ and a probit analysis. These analyses agreed in showing no change as a result of the heterokaryotic association. Also in agreement was an analysis of variance of the angular transformed percentage survivals which is given below.

$\begin{array}{lcc}\text { Item } & \begin{array}{c}\text { Degrees of } \\ \text { freedom }\end{array} & \begin{array}{c}\text { Mean } \\ \text { square }\end{array} \\ \text { (1) Conc. of poison } & 2 & 705 \cdot 2 \\ \text { (2) 'Before' vs. 'After' } & 1 & 10 \cdot 4 \\ 1 \times 2 & 2 & 5 \cdot 2 \\ \text { Duplicates } & 24 & 18 \cdot 2\end{array}$

The other component of the heterokaryon, homokaryon 2 . w, also showed no change in adaptability following its association with homokaryon 1.5 and indeed this was the general finding from such comparisons. It would seem, therefore, that the differences in adaptability between these homokaryon clones are characteristically nuclear in origin.

\section{Differences within homokaryon clones}

The results in Table 1 show that there was considerable heterogeneity in the ability of asexual spores of uniform age, and produced by single homokaryon colonies to adapt, i.e. survive and grow, at different concentrations of mercuric chloride. There is also heterogeneity, although less marked, in the ability of similar samples of asexual spores to adapt to new sugars (Briault, 1956).

These results, however, by no means convey the full extent of this heterogeneity among single asexual spores, for even among those spores which could adapt to the new conditions there occurred considerable variation in the duration of the initial lag period. There is, of course, some variation in the onset of germination among single asexual spores even on the normal medium, but this is regularly increased up to fourfold on the adaptive media.

Direct evidence of heterogeneity between the vegetative hyphae of single homokaryon colonies was more difficult to obtain, mainly because propagations by single vegetative hyphae resulted in mechanical damage to the colony and the transferred cells. As a consequence it was not always easy to separate failure to adapt, which led to death, from death resulting from mechanical damage.

One approach has been to investigate the effect of the number of hyphal growing tips in an inoculum on their survival and on duration and variation in the lag period on mercuric chloride. As a control, to determine effects due to the experimental procedure, duplicate inocula were placed on the normal medium and their performance used to correct the results obtained on the poison.

In general, inocula containing fifty or more intact growing-tips always successfully adapted to quantities of poison as high as 5 p.p.m. after a uniform lag period of 3-4 days, the details varying between different homokaryon clones. With smaller inocula there were frequent failures to adapt as well as variation in the duration of the lag period of those which did adapt. An 
example of the type of results obtained is given in Table 4. These relate to transfers of homokaryon 2.10 from the normal medium to 5 p.p.m. of mercuric chloride.

Table 4. The effect of inoculum size on mean lag periods

$\begin{array}{cccc}\begin{array}{c}\text { Number of intact } \\ \text { growing tips in } \\ \text { inoculum }\end{array} & \begin{array}{c}\text { Normal } \\ \text { medium }\end{array} & \begin{array}{c}\text { L p.p.m } \\ \text { Mean lag in days }\end{array} & \begin{array}{c}\text { Lag due to } \\ \text { poison }\end{array} \\ 4 & 5 \cdot 6 & 17 \cdot 0 & 11 \cdot 4 \\ 16 & 2 \cdot 9 & 12 \cdot 5 & 9 \cdot 6 \\ 32 & 2 \cdot 1 & 9 \cdot 6 & 7 \cdot 5 \\ 50-200 & 2 \cdot 0 & 5 \cdot 3 & 3.3\end{array}$

Not only had the mean lag period, after correction, more than trebled from the largest to the smallest inoculum, but the variation in the lag periods of replicate inocula increased fivefold over the same range. More important, however, while all inocula with more than 32 intact tips adapted, 6 out of 13 and 6 out of 15 of the 4 and 16 tip inocula failed to grow. Pooling the results from these two sizes of inoculum, 12 grew and 2 failed on the normal medium while 4 grew and 10 failed on the poison. On both media deaths will have occurred from mechanical damage and other upsets resulting from the experimental procedure, but the significant difference $(P=0.01-0.001)$ between the frequency of deaths on the two media must be specifically related to the inability of some undamaged cells to adapt to the presence of the poison. Such evidence can only imply differences between the vegetative hyphae of single homokaryon colonies in their ability to adapt to the new medium.

An analysis of the origin of this heterogeneity between both single asexual spores and vegetative hyphae from individual homokaryon colonies is described in later sections where the former variation is used as the basis of selection experiments.

\section{Selection for adaptability to the presence of mercuric chloride}

It was shown (Jinks, 1957) that there was variation in morphological characters among the asexual progeny of single homokaryon colonies, and that selection for the extreme variants could give rise to large differences in phenotype depending solely on differences in cytoplasmic content. Similar selection has been used to investigate the variation in adaptability between colonies derived from the asexual spores of individual homokaryon colonies.

Each cycle of selection was initiated by raising large numbers of sister colonies on the normal medium each from a single asexual spore produced by a single parent homokaryon on the same medium. Five of these sister colonies were chosen at random and samples of their asexual spores simultaneously tested for their ability to adapt to two or more levels of mercuric chloride. In the first cycle the two colonies, whose asexual spores gave the highest and lowest percentage survival, respectively, were retained for the second cycle. In the second, and subsequent cycles, the asexual spores of the two sets of 
five colonies were simultaneously tested and only one from each set retained, namely, the highest of the five from the high line and the lowest of the five from the low line. An unselected control line was maintained in the same way, except that in each cycle one colony was chosen at random out of the five tested instead of on the basis of the performance of its asexual spores on the poison. No colony in the direct line of selected descent was exposed to the poison: their change cannot, therefore, be referred to a direct action of the poison. Selection was in fact practised for adaptability rather than adaptation.

A typical example is the selection for high- and low-percentage adaptation to mercuric chloride of the asexual spores of homokaryon 1.5. Selection was here carried out for four cycles at two or three concentrations of mercuric chloride, chosen so that one lay on either side of the $50 \%$ survival point. To achieve this it was necessary to adjust the concentrations of mercuric chloride used in successive cycles, basing the adjustments on performance in the previous cycle. All the concentrations used, however, fell within the range 2-5 p.p.m. The final comparison of the selections was made at 5 different concentrations of mercuric chloride, ranging from 0 to 5 p.p.m.

The estimated LD 50 values for the high and low selections and the control line are given in Table 5 .

Table 5. The LD 50 values in p.p.m. of mercuric chloride for the high and low selections and the control line derived from homokaryon $1 \cdot 5$

\begin{tabular}{|c|c|c|c|c|c|}
\hline \multirow{2}{*}{$\begin{array}{l}\text { Cycles of } \\
\text { selection }\end{array}$} & \multirow[t]{2}{*}{ Parent: } & $\mathbf{1}$ & $\mathbf{2}$ & $\mathbf{3}$ & 4 \\
\hline & & \multicolumn{4}{|c|}{ LD 50 value, in p.p.m. mercuric chloride } \\
\hline High line & - & $3 \cdot 17$ & $4 \cdot 43$ & $4 \cdot 34$ & $4 \cdot 62$ \\
\hline Control line & 3.62 & $3 \cdot 13$ & $\mathbf{3 \cdot 5 5}$ & $4 \cdot 03$ & $3 \cdot 70$ \\
\hline Low line & - & $3 \cdot 28$ & $3 \cdot 65$ & $3 \cdot 81$ & $3 \cdot 04$ \\
\hline
\end{tabular}

The results in Table 5 show that marked progress was made after only four cycles of selection; a highly significant difference of $1.52 \pm 0 \cdot 18$ was achieved between the high and low lines. Moreover, taking the control rather than the parental value as a base line, progress was made in both directions.

Two attempts were made to estimate the heritability of this character during the course of the selections. The first was made between the first and second cycles when the colonies chosen to continue the selections were supplemented by others chosen at random. In all, eleven colonies from the first cycle were used as parents, three from each of the selections and five from the control. The correlation coefficient between the performance of parent colonies in the first cycle and the mean performance of their daughter colonies in the second was not significant $(r=\mathbf{0 \cdot 3 3})$. When, however, we average the performance within each of the selection lines and within the control line the parent/ daughter correlation is $\mathbf{0 . 9 3}$. Repeats on a smaller scale for the second and third and third and fourth cycles gave correlations of 0.98 and 0.66 , respectively, the former being highly significant.

The second estimate was a comparison between the difference in perfor- 
mance of the parents chosen to continue the high and low selections (the selective differential) and the observed difference in performance of random samples of the asexual spores of five of their daughter colonies in the next cycle. Over the four cycles of selection the regression of observed difference on the selective differential was $0.96 \pm 0 \cdot 36$, the expected value being 1.00 if all the differences between parent colonies were heritable.

The mean lag period of those transfers which successfully adapted was scored throughout the selections and the results for the highest concentration of poison used in each cycle are given in Table 6. Some of the variation between cycles for this character was undoubtedly due to the deliberate variations in the concentrations of poison used (as described earlier), but within each cycle the comparison between lines was independent of this variation.

Table 6. The mean lag period in days of the asexual spores of the high, low and control lines of homokaryon 1.5 which successfully adapted to the highest concentrations of poison used in each cycle

\begin{tabular}{lllrr}
$\begin{array}{c}\text { Cycles of } \\
\text { selection : }\end{array}$ & 1 & \multicolumn{2}{c}{$\begin{array}{c}3 \\
\text { Mean lag in days }\end{array}$} \\
\cline { 4 - 5 } High line & $4 \cdot 86$ & $2 \cdot 99$ & $3 \cdot 84$ & $2 \cdot 78$ \\
Control line & $4 \cdot 55$ & $2 \cdot 55$ & $3 \cdot 96$ & $4 \cdot 87$ \\
Low line & $4 \cdot 25$ & $5 \cdot 42$ & $6 \cdot 21$ & $6 \cdot 31$
\end{tabular}

Clearly, the two estimates of the adaptabilities of the selection lines, namely, their LD 50 values (Table 5) and their mean lag periods (Table 6) are highly negatively correlated and hence provide much the same picture of the progress of selection. The negative correlation is general throughout the data, being present in every individual sample of five sister colonies scored during the selections. But, as might be expected with such small samples, the negative correlations are significant only in four of them.

The two homokaryons re-extracted in the form of asexual spores of the heterokaryons between colonies of the high and low selections no longer showed the difference in adaptability to mercuric chloride. This is shown by the following analysis of variance of the angular transformed percentage adaptations of asexual spores taken from the re-extracted homokaryons when plated on 3 and 5 p.p.m. of the poison.

\section{Item}

(1) Differences between homokaryons after re-extraction from heterokaryon

(2) Concentrations of poison

(3) Replicate concentrations of poison $1 \times 2$

$1 \times 3$

\section{Degrees of freedom}

1

1

2

$\mathbf{1}$

$$
\begin{gathered}
\text { Mean } \\
\text { squares } \\
\mathbf{3 4 \cdot 9 9} \\
\\
\mathbf{6 4 1 \cdot 1 8} \\
\mathbf{1 0 \cdot 7 9} \\
\mathbf{3} \cdot 42 \\
\mathbf{3 8} \cdot 14
\end{gathered}
$$$$
4.641 \cdot 18
$$

None of the highly significant differences in adaptability survived the co-existence in the heterokaryon state. These differences must, therefore, be cytoplasmic in origin.

Further heterokaryons were made between colonies of the high or low 
selections and unselected colonies of independent clones, e.g. homokaryon 2.6. The $\mathrm{LD} 50$ values of the asexual spores of the homokaryons re-extracted from the heterokaryons in one of these experiments are listed in Table 7. For comparison the LD 50 values of the selection lines before heterokaryon formation are listed in the first column of this Table.

Table 7. The effect of heterokaryotic association on the $L D 50$ values of the high and low selections of homokaryon 1·5 and a normal colony of homokaryon 2.6

LD 50 values (p.p.m. of mercuric chloride) and

Heterokaryon

High selection + homokaryon 2.6

Low selection + homokaryon 2.6 their standard errors

\begin{tabular}{|c|c|c|}
\hline \multicolumn{2}{|c|}{ Selection lines of homokaryon 1.5} & \multirow{2}{*}{$\begin{array}{c}\text { Normal } \\
\text { homokaryon 2.6 } \\
\text { After } \\
\text { heterokaryon }\end{array}$} \\
\hline $\begin{array}{c}\text { Before } \\
\text { heterokaryon }\end{array}$ & $\begin{array}{c}\text { After } \\
\text { heterokaryon }\end{array}$ & \\
\hline $\begin{array}{l}4 \cdot 62 \pm 0 \cdot 12 \\
3 \cdot 04 \pm 0 \cdot 19\end{array}$ & $\begin{array}{l}3 \cdot 92 \pm 0.05 \\
3 \cdot 67 \pm 0.06\end{array}$ & $\begin{array}{l}4.21 \pm 0.05 \\
4.02 \pm 0.06\end{array}$ \\
\hline
\end{tabular}

The difference between the LD 50 values of the selection lines and homokaryon 2.6 after extraction from the heterokaryons must be under nuclear control. The lowering of the LD 50 values of the high selection and the raising of that of the low selection so that both approach the value for the control line $(3 \cdot 63 \pm 0 \cdot 07)$ must, on the other hand, be attributed to exchanges between the selected and wild-type cytoplasms while in the heterokaryons. A small part $(16 \%)$ of the difference in LD 50 between the high and low selections has, however, survived heterokaryotic association with the normal cytoplasm of homokaryon 2.6. Similarly, a difference of the same magnitude and sign has arisen between the two samples of homokaryon 2.6 following their association with the high and low selections in the heterokaryons. These persistent differences between the selection lines and the new differences within homokaryon 2.6 must be related to cytoplasmic differences between the two heterokaryons, which in turn could only have been introduced by their noncommon component, namely the selected lines of homokaryon 1.5.

Attempts to select for differences in adaptability to mercuric chloride in homokaryon 2.6 failed where the procedures were identical with those just described for homokaryon 1.5. Modification of the procedures so as to increase the selection pressure might well have produced a response in homokaryon 2.6. But this would have upset one of our aims, namely to compare the two genotypes of homokaryons 1.5 and 2.6 under identical conditions of selection. A number of experiments described later demonstrate conclusively that cytoplasmically differentiated lines can be obtained within homokaryon 2.6, which differ significantly in the ability of their asexual spores to adapt to mercuric chloride.

\section{Selection for adaptability to new sugars}

We may recognize two types of difference in adaptability to new sugars between samples of asexual spores taken from sister colonies of a homokaryon clone. Either of these differences, which will be referred to as general and 
specific, might be utilized as the basis of a selection experiment. General adaptability refers to the mean adaptability of a sample of asexual spores when tested on a number of new sugars. Specific adaptability, on the other hand, refers to an above or below average performance of a particular sample of asexual spores on a particular new sugar. We can illustrate this distinction by reference to tests of the asexual spores of five asexually-derived sister colonies of homokaryon 2.6 on the four sugars, galactose, arabinose, xylose and lactose. The results, given in Table 8, are the numbers of normal colonies, produced by the five samples of asexual spores when equally divided between the four sugars.

Table 8. The totals of successful adaptations when samples of asexual spores from five sister colonies of homokaryon $\mathbf{2 . 6}$ on the normal medium were equally divided between media containing four new sugars

$\begin{array}{ccccccc}\text { Colony } & \text { Galactose } & \text { Arabinose } & \text { Xylose } & \text { Lactose } & \text { Total } & \begin{array}{c}\text { Expected } \\ \text { totals }\end{array} \\ 1 & 292 & 272 & 328 & 137 & 1029 & 902 \\ 2 & 160 & 204 & 224 & 66 & 654 & 943 \\ 3 & 142 & 214 & 182 & 47 & 585 & 560 \\ 4 & 254 & 326 & 341 & 168 & 1089 & 964 \\ 5 & 169 & 147 & 183 & 52 & 551 & 539 \\ \text { Totals : } & 1017 & 1163 & 1258 & 470 & 3908 & \end{array}$

Differences in general adaptability were detected by comparing the observed and expected row totals in Table 8 by a $\chi^{2}$ for 4 D.F., the expected totals being estimated from the relative number of asexual spores in each of the samples taken from the five colonies.

By using the observed column and row totals we can test for heterogeneity between the performance of these samples on the four sugars, i.e. for differences in specific adaptability, by calculating the interaction $\chi^{2}$ for 12 D.F.

Analysis of the data in Table 8 gives the following results:

\begin{tabular}{lrrc} 
& \multicolumn{1}{c}{$\chi^{2}$} & N & $P$ \\
Specific adaptability & $\mathbf{5 2 . 5 7}$ & 12 & $<0.001$ \\
General adaptability & 124.05 & 4 & $<0.001$
\end{tabular}

Clearly, we have both specific and general differences in adaptability between the five sister colonies of homokaryon 2.6. A major contribution to the differences in specific adaptability between the five colonies comes from the tests on the two sugars xylose and arabinose, the asexual spores of some colonies (e.g. 1 and 5 in Table 8) adapting more readily to xylose while those of other colonies show no preference or a slight one for arabinose. A selection experiment was, therefore, initiated in homokaryon 2.6 where the high line was for high adaptability to arabinose and low to xylose, and the low line high adaptability to arabinose and low to xylose. General adaptability, measured as the mean adaptability to both sugars, was scored as a secondary character. The actual selective procedures were those described earlier for adaptability to mercuric chloride. 
There was a marked, although asymmetrical, response to selection. Thus in the parent clone xylose was already at an advantage as an alternative sugar $(X \mid A=1 \cdot 21)$ and the high selection only raised this to $1 \cdot 29$. The low selection, however, produced a greater response and over three cycles, the value of $X / A$ fell to $\mathbf{0 \cdot 8 4}$.

General adaptability was correlated with the ratio $X / A$. In the first cycle of selection this correlation was as high as $\mathbf{- 0 \cdot 8 7}$, suggesting that only colonies with high general adaptability could adapt to arabinose as well as they did to xylose. This negative correlation persisted throughout, although it reached a value as low as $\mathbf{- 0 . 2 2}$ in the sample of asexual spores taken from the colonies of the third cycle of selection.

Mean lag period, the other metric for assessing differences in adaptability, was scored throughout the selections. In the parent clone the mean lag on xylose was 4.56 days shorter than that on arabinose. In the high line this advantage in mean lag was raised to $\mathbf{5 \cdot 3 6}$ days while in the low line it was reduced to 3.99 days. Thus this metric also shows a response to selection for differences in adaptability to xylose and arabinose.

Neither significant general nor specific differences in adaptability to the four new sugars were detected in three successive samples of five sister colonies of homokaryon 1.5; hence attempts to select within this clone were abandoned.

\section{Adaptability to mercuric chloride and the new sugars}

The differences between the selections for high and low adaptability to mercuric chloride and to the new sugars are solely cytoplasmic in origin. We may now inquire whether the cytoplasmic systems involved are common to both the adaptability to the poison and to the sugars or whether the two are independent. Direct evidence on this point can be obtained by scoring adaptability to both the poison and the sugars on the same samples of asexual spores, the latter being taken either from random samples of sister colonies or from selections for adaptability to one or other of the environmental changes.

Five sister colonies of homokaryon 2.6 were chosen to give a highly significant difference both in general and specific adaptability to the sugars $(P<0.001$ for both). Simultaneous with the tests on the sugars, asexual spores of the same samples were also tested on two levels of mercuric chloride, viz. 3 and 5 p.p.m. Analyses revealed no significant differences in adaptability to the poison. An identical result was obtained by using the mean lag periods on the sugars and poison as the metric. Again, there were highly significant differences between the five colonies on the sugars $(P<0.001)$ but not on the poison. These, and similar findings from other experiments, leave little doubt that adaptability to the poison and sugars have independent cytoplasmic origins. This is confirmed in the next section.

\section{The adaptive and morphological systems}

It was observed (Jinks, 1957) that variation in rate of growth, perithecial density, pigmentation, etc., was highly correlated and apparently controlled 
by the same cytoplasmic system. Whether this system is independent of those controlling adaptability to the poison and the sugars was now examined.

One way of making this comparison is to score the rate of growth of the lines selected for differences in adaptability. As an example, five colonies were chosen at random from the high, low and control lines of homokaryon 1.5 after 4 cycles of selection for differences in adaptability to mercuric chloride. The experiment was replicated four times by single asexual spore transfers and each replicate duplicated by mass hyphal transfers; all colonies, amounting to 120 , were grown both on the normal medium and on 4 p.p.m. of the poison.

Analyses of the total growth over three successive days showed that significant differences in rate of growth had arisen both within and between the selection and control lines.

$\begin{array}{lccc}\text { Item } & \begin{array}{c}\text { Degrees of } \\ \text { freedom }\end{array} & \begin{array}{c}\text { Normal } \\ \text { mean squares }\end{array} & \begin{array}{c}\text { M p.p.m. } \mathbf{H g C l}_{\mathbf{2}} \\ \text { mean squares }\end{array} \\ \text { Between lines } & 2 & 4 \cdot 13 & \mathbf{1 6 \cdot 2 7} \\ \text { Within lines } & 12 & 3 \cdot 83 & \mathbf{2 \cdot 4 1} \\ \text { Replicates } & 45 & 1 \cdot 22 & 0 \cdot 89 \\ \text { Duplicates } & 60 & 0 \cdot 29 & 0 \cdot 41\end{array}$

The five colonies chosen to represent each line were also scored for the percentages of their asexual spores which were capable of adapting to 4 p.p.m. of the poison and the correlation between this and their rates of growth was determined (Table 9).

Table 9. Correlations between percentage adaptation of asexual spores on 4 p.p.m. mercuric chloride and the growth rate on the normal medium +4 p.p.m. mercuric chloride for the selections and control line of homokaryon 1.5

\begin{tabular}{lrr} 
Line & \multicolumn{2}{c}{ Medium } \\
High & $\begin{array}{r}\text { Normal } \\
\text { Ho.48.m. }\end{array}$ \\
Control & -0.43 & +0.06 \\
Low & +0.28 & -0.71 \\
Overall & 0.00 & +0.35 \\
& & -0.09
\end{tabular}

None of the correlations in Table 9 is significant.

A similar result was found by comparing the adaptabilities of lines selected for differences in rate of growth. In some cases these selections affected the ability of their asexual spores to adapt to mercuric chloride. But results such as those given in Table 10, which were obtained from three selections for fast and slow growth in homokaryon $2 \cdot 6$ show that the correlation between rate of growth and the ability to adapt to the poison must be low.

In other cases selections for fast and slow growth left adaptability unaffected. Such an example is provided by further selections of homokaryon 2.6, three slow and two fast, with rates of growth as different as 1.2 and $\mathbf{2 . 4} \mathrm{mm}$./day, respectively. Their adaptive properties were tested on both 3 and 5 p.p.m. of the poison. There were no differences in adaptability between selections 
$(P=0.95)$ and a doubtfully significant difference within them $(P=0.05)$. As a consequence the correlations with rate of growth were not significant, being $\mathbf{0 . 2 4}$ and $-\mathbf{0 . 4 0}$ for the two concentrations of poison.

Table 10. The percentage adaptation and mean lag of random samples of asexual spores of three independently derived fast and slow selections of homokaryon 2.6

\begin{tabular}{|c|c|c|}
\hline Selection & $\begin{array}{l}\text { Percentage adaptation } \\
\text { to } 3 \text { p.p.m. } \mathrm{HgCl}_{2}\end{array}$ & $\begin{array}{l}\text { Mean lag in days } \\
\text { on } 3 \text { p.p.m. } \mathrm{HgCl}_{2}\end{array}$ \\
\hline Fast & $\begin{array}{l}20.4 \\
19.3 \\
14.0\end{array}$ & $\begin{array}{l}5 \cdot 73 \\
6 \cdot 04 \\
4 \cdot 89\end{array}$ \\
\hline Mean & $17 \cdot 9$ & $5 \cdot 55$ \\
\hline Slow & $\begin{array}{r}38 \cdot 5 \\
3 \cdot 8 \\
2 \cdot 7\end{array}$ & $\begin{array}{l}4 \cdot 26 \\
7 \cdot 34 \\
9 \cdot 30\end{array}$ \\
\hline Mean & $15 \cdot 0$ & 6.97 \\
\hline
\end{tabular}

In contrast, adaptability to the new sugars was in general more highly correlated with rate of growth. For example, analysis of the adaptabilities to the four sugars of the fast and slow selections of homokaryon 2.6 showed highly significant general and specific differences $(\boldsymbol{P}<\mathbf{0 . 0 0 1}$ for both), the correlation between rates of growth and general adaptability being $\mathbf{0 . 9 0}$ $(P=0.05-0.02)$. The same high correlation also applied to specific adaptability, the best of the four sugars for the fast selection being consistently galactose and for the slow selection, lactose.

The other metric used for adaptability, lag period, gave similar results, the mean lag on the four sugars of the fast and slow selections being $5 \cdot 65$ and $\mathbf{7 \cdot 3 5}$ days, respectively. A comparison of the rates of growth and the mean lag on all sugars gave a high negative correlation of $\mathbf{- 0 \cdot 7 2}$.

\section{The cytoplasmic systems}

Before discussing the contribution of the cytoplasm to variation in adaptability to new media it is worth noting that no nuclear contribution to intra-clonal variation for this character was found during extensive testing. Nevertheless, the same testing techniques have shown (see Differences between homokaryon clones) that the differences between the parental clones for this character were, as one might expect, characteristically nuclear in origin. The phenotypic differences produced by the latter may be as large as any which can be achieved by variation in the cytoplasmic systems and they appear to exert considerable influence on the outcome of the selections for differences of cytoplasmic origin; so much so, that the nuclear system might be formally described as epistatic to the cytoplasmic system involved in the adaptive changes.

The cytoplasmic systems involved in the differences in adaptability have the same general properties as those responsible for the variation in rate of growth, perithecial density and pigmentation, which arise during differentia- 
tion and ageing (Jinks, 1956). Thus they produce continuous variation in the adaptability of sister colonies derived from single asexual spores or single vegetative cells. From the behaviour under selection it is clear that these differences persist in asexual propagation and can be accumulated to give smooth changes in phenotype. The absence of segregation for quantitative differences in adaptability in the asexual progenies of single colonies both before and during the heterokaryon tests suggests that major cytoplasmic mutations are not involved in the variation, which probably, therefore, depends solely on differences in the equilibria of normal cytoplasmic constituents (Arlett, 1957; Jinks, 1958).

An important feature of the phenotypic variation in morphology that arose from cytoplasmic changes during differentiation and ageing was the high correlation between all the characters followed: all changed when any one was selected. Whether, however, this was true pleiotropy or sprang from mechanical association of the determinants in transmission could not be decided.

In the present work lines selected for differences in adaptability to new media showed significant differences for the other characters followed. Nevertheless, the correlations between the selected and unselected characters are in general quite low. This is particularly true of the homokaryon $\mathbf{1 . 5}$ lines selected for differences in adaptability to mercuric chloride, where the overall correlations with rate of growth were virtually zero (Table 9 ). The same is true of the reverse selections for homokaryon 2.6, where lines showing the maximum differences in rate of growth, obtainable by selection, showed no consistent differences in adaptability to mercuric chloride.

The correlations between rate of growth and adaptability to the new sugars are quite high $(r=0.8-0.9)$ and there is every indication that in general the more vigorously a colony grew on the normal medium the greater the adaptability of its asexual spores to the new sugars. In view of this difference in relationship between rate of growth and adaptability to the new sugars, on the one hand, and rate of growth and adaptability to mercuric chloride on the other, it seems clear that adaptability to the new sugars must be independent of adaptability to mercuric chloride.

While our primary concern was with cytoplasmic inheritance, of which adaptability to new media is just one more easily demonstrable example, our results, nevertheless, have a bearing on the nuclear basis of adaptation.

The importance of the nuclear component, which we have suggested is epistatic to all others, has been illustrated by clonal differences for the ability of asexual spores and vegetative cells to adapt to new media. But we have also shown that possession of the right nuclear genes by a spore or cell is not in itself sufficient to ensure successful adaptation. A cytoplasmic component is also required and spores and vegetative cells may be deficient or unbalanced for this component.

Adaptation is thus basically the selection of those cells, spores or colonies which possess a nuclear-cytoplasmic combination capable of producing the necessary re-adjustments required for successful growth in the new environment. And although in the wild state this selection would be automatically 
applied by the new environment itself, conscious selection in the old environment, as described in this paper, can significantly affect the chances of survival on exposure to a new one.

\section{REFERENCES}

ARLETt, C. F. (1957). Induction of cytoplasmic mutations in Aspergillus nidulans. Nature, Lond. 179, 1250.

Briault, P. G. (1956). Cytoplasmic changes associated with adaptation and differentiation. Nature, Lond. 178, 1223.

Gibson, A. \& Griffin, D. M. (1958). Cytoplasmic variation in Nectria stenospora. Nature, Lond. 181, 1222.

Jinks, J. L. (1952). Heterokaryosis; a system of aduptation in wild fungi. Proc. Roy. Soc. B, 140, 83.

JiNKs, J. L. (1954). Somatic selection in fungi. Nature, Lond. 174, 409.

JiNKs, J. L. (1956). Naturally occurring cytoplasmic differences in fungi. C.R. Lab. Carlsberg, (Ser. Physiol.) 26, 183.

Jinks, J. L. (1957). Selection for cytoplasmic differences. Proc. Roy. Soc. B, 146, 527.

Jinks, J. L. (1958). Cytoplasmic differentiation in fungi. Proc. Roy. Soc. B, 148, 314.

Ponteconvo, G. (1953). The genetics of Aspergillus nidulans. Advanc. Genet. 5, 141.

(Received 9 September 1958) 